\title{
Heterotic Effect of Some Intra-specific Crosses of Brinjal (Solanum melongena L.) for Yield and Yield Attributes
}

\author{
Niranjan Kumar Chaurasia ${ }^{1}$, Prabalee Sarmah ${ }^{2}$, Nagendra Sarma Barua ${ }^{2 *}$, \\ Debojit Sarma ${ }^{2}$, Hiramani Barman ${ }^{2}$ and Purna Kanta Barua ${ }^{2}$ \\ ${ }^{1}$ Department of Plant Breeding and Genetics, Bihar Agricultural University, \\ Sabour, Bihar, India \\ ${ }^{2}$ Department of Plant Breeding and Genetics, Assam Agricultural University, \\ Jorhat-785013, Assam, India \\ *Corresponding author
}

\begin{tabular}{|c|}
\hline Keywords \\
\hline $\begin{array}{l}\text { Brinjal, hybrid } \\
\text { vigour, mid-parent } \\
\text { heterosis, better- } \\
\text { parent heterosis and } \\
\text { standard-parent } \\
\text { heterosis }\end{array}$ \\
\hline Article Info \\
\hline $\begin{array}{l}\text { Accepted: } \\
15 \text { November } 2019 \\
\text { Available Online: } \\
10 \text { December } 2019\end{array}$ \\
\hline
\end{tabular}

\section{Introduction}

Brinjal (Solanum melongena L., $2 \mathrm{n}=24$ ), also known as eggplant or aubergine, is an important Solanaceous vegetable crop. It is widely grown in India throughout the year.
Eighteen $\mathrm{F}_{1} \mathrm{~s}$ were generated from line $\mathrm{x}$ tester mating design using six parents as lines and three parents as testers during rabi, 2015-16. The $\mathrm{F}_{1} \mathrm{~s}$ along with their parents were evaluated altogether during rabi, 2016-17 for fruit yield and component characters in a randomized block design (RBD) with three replications at the Experimental Farm, Department of Horticulture, Assam Agricultural University, Jorhat, Assam. The heterosis was worked out for the quantitative traits viz., days to 50 percent flowering, plant height $(\mathrm{cm})$, number of branches per plant, fruit length $(\mathrm{cm})$, fruit girth $(\mathrm{cm})$, fruit weight $(\mathrm{g})$, number of fruits per plant, number of seeds per fruit, fruit yield per plant $(\mathrm{kg})$, crude fiber content $(\%)$ and shelf life (days) of fruits. The magnitudes of mid-parent heterosis (MPH), better-parent heterosis (BPH) and standard heterosis (SPH) were obtained in all the eighteen $\mathrm{F}_{1}$ s for eleven characters. The study revealed that the $\mathrm{F}_{1} \mathrm{SX} \times \mathrm{SM}-6-7$ gave the highest estimate of SPH $(40.17 \%)$ and it was followed by the other promising $\mathrm{F}_{1}$ 's viz., $\mathrm{BM} \times \mathrm{SM}-6-7$ (39.19\%), $\mathrm{SX} \times$ Longai (36.61\%), $\mathrm{DH} \times$ Longai $(36.12 \%)$, SX $\times$ JC-1(35.38\%), MLC-1 $\times$ JC-1 $(33.66 \%)$, MLC-1 $\times$ Longai $(30.59 \%)$ and $\mathrm{BM} \times$ Longai $(25.80 \%)$ for fruit yield per plant. 
wide range of fruit shapes and colours, ranging from oval or egg-shaped to long clubshaped; and from white, yellow, green through various degrees of purple pigmentation to almost black fulfils the demand of local people. It is not possible to have one common cultivar to suit different localities, climates and local preferences. Locally adaptable varieties have many important traits including yielding ability that could elevate the performance of the improved varieties when combined together by the process of hybridization. Hence, keeping this point in view, the present research work was undertaken with an objective to evaluate the brinjal $F_{1} s$ for their heterotic performance in respect of various important characters.

\section{Materials and Methods}

The present study was conducted at Experimental Farm, Department of Horticulture, Assam Agricultural University, Jorhat, Assam. The $\mathrm{F}_{1}$ hybrids were generated during rabi, 2015-16 using six lines namely, Utsav, Dari Hariharka, Manipur local-3, Manipur local-1, Baromohiya and Sagoli Xingiya and three testers viz., JC-1, SM-6-7 and Longai by hand emasculation and pollination. The seeds of eighteen $\mathrm{F}_{1} \mathrm{~S}$ and their nine parents were sown in the nursery bed and one-month-old seedlings were transplanted in the main field with spacing 75 $\mathrm{cm} \times 60 \mathrm{~cm}$ in an RBD with three replications during rabi, 2016-17. Each plot of size $3 \mathrm{~m} \mathrm{x}$ $3 \mathrm{~m}$ comprised five plants in a row and four rows in a plot. The observations were recorded on five competitive plants selected at random from each plot on plant height $(\mathrm{cm})$, number of branches per plant, fruit length $(\mathrm{cm})$, fruit girth $(\mathrm{cm})$, fruit weight $(\mathrm{gm})$, number of fruits per plant, number of seeds per fruit, fruit yield per plant $(\mathrm{kg})$, crude fiber content on fresh weight basis (per cent) and shelf life of fruits on ordinary laboratory temperature (days). The counting of days to 50 per cent flowering was done on a plot basis. The shelf life of fruit was calculated on the basis of physiological loss of weight using the formula referred by Murugan et al., (2011). The crude fibre content was calculated by using the protocol used for the estimation of crude fibre by Sadasivam and Manickam (1992). Critical difference was computed as SEd x t-value at error degree of freedom at 5\% level of significance. Per cent estimates for MPH, $\mathrm{BPH}$ and $\mathrm{SPH}$ and their corresponding values of standard errors were calculated as per standard procedures.

\section{Results and Discussion}

The analysis of variance revealed highly significant differences among the entries including $F_{1} s$ and their parental lines for most of the yield contributing traits indicating that parental lines have generated sufficient variability in the materials under study (Table 1). The extent of per cent mid-parent heterosis, better-parent heterosis and standardparent heterosis for various traits contributing to yield and quality are presented in Table $2 \mathrm{a}$, $2 \mathrm{~b}, 2 \mathrm{c}$ and 3 . The promising crosses showing significant standard parent heterosis for fruit yield and component characters along with their mean performances are also presented in Table 3. In vegetables, earliness is more desirable as it fetches a higher price and fits well with multiple cropping systems (Kamalakannan et al., 2007). In the present study, the $\mathrm{F}_{1} \mathrm{~s}$ Utsav x JC-1, DH x JC-1, MLC$3 \mathrm{x}$ Longai and Utsav x SM-6-7 expressed significant and desirable mid-parent heterosis, better-parent heterosis and standard heterosis for days to 50 per cent flowering. These $F_{1} s$ also exhibited the significant and favourable heterosis for the number of branches per plant which is desirable for production of more number of fruits and thereby higher yield in brinjal. This finding was also supported by Joshi et al., (2008), Chowdhury et al., (2010), Singh et al., (2012), Biswas et al., (2013), 
Dudhat et al., (2013), Makani et al., (2013), Dubey et al., (2014) and Desai et al., (2016).

In brinjal, the ideal plant type should be one which is tall and supports yield and its component traits. The data on heterosis support that some of the crosses, in general, were taller whereas some were shorter than their standard parent. The $\mathrm{F}_{1} \mathrm{BM} \times \mathrm{JC}-1$ was found to be tallest over MP, BP and SP whereas the $\mathrm{F}_{1} \mathrm{BM} \times \mathrm{SM}-6-7$ was the tallest of the $\mathrm{F}_{1} \mathrm{~s}$ over both mid-parent and the better parent. These two $F_{1} s$ also had heterotic effects for the number of branches per plant and number of fruit per plant which is directly correlated to yield and its component traits. Similar findings have also been reported by Suneetha et al., (2008), Pachiyappan et al., (2012), Biswas et al., (2013), Makani et al., (2013), Reddy and Patel (2014) and Dubey et al., (2014). The shortest plant stature is necessary where the problem of lodging is prevalent. To overcome this problem, we need to search for short stature variety/hybrid which would compromise with yield since plant height is positively correlated with yield. The $\mathrm{F}_{1}$ MLC-3 x JC-1 has the shortest plant height over their MP and BP whereas $\mathrm{F}_{1}$ Utsav $\mathrm{x}$ JC1 had shorter plant height over SP but was observed to be lower yielder.

The number of branches per plant is a major parameter which can support yield and its component traits. In the present study, parental lines Baromohiya and SM-6-7 had the highest number of primary branches among lines and testers, respectively. The cross combination DH x SM-6-7 recorded the highest positive heterosis over MP, BP and SP. The cross DH $x$ Longai had a heterotic effect over MP and $\mathrm{BP}$ while the $\mathrm{F}_{1} \mathrm{BM} \times \mathrm{SM}-6-7$ had heterosis over only SP for this trait. Studies on MPH, $\mathrm{BPH}$ and $\mathrm{SPH}$ in different sets of material were reported by earlier workers viz., Shafeeq et al., (2007), Joshi et al., (2008), Sharma (2010) and Reddy and Patel (2014).
Fruit length and fruit girth are important parameters for deciding consumer preference. Therefore, the crosses showing negative heterosis for fruit length might be useful as shorter fruits are preferred in a certain region of India. The cross DH x JC-1 exhibited negative heterosis over the MP, BP and SP for fruit length. Majority of the crosses showed negative heterosis over SP for this trait.

Similar studies were conducted on different materials by Shafeeq (2005), Suneetha et al., (2008) and Chowdhury et al., (2010). Similarly, in certain other regions of India, mainly long fruit with medium girth is preferred. In this regard, the $\mathrm{F}_{1} \mathrm{BM} \times \mathrm{SM}-6-7$ showing significant positive estimates for $\mathrm{MPH}$ and $\mathrm{BPH}$ and the $\mathrm{F}_{1} \mathrm{BM} \times \mathrm{JC}-1$ showing SPH over JC-1 can be exploited for the hybrid breeding programme. Positive heterosis at different levels for fruit length was earlier reported by Pachiyappan et al., (2012), Biswas et al., (2013), Dubey et al., (2014), Makani et al., (2013), Reddy and Patel (2014) and Venkata et al., (2014).

Fruit weight is one of the important component traits having a positive correlation with the fruit yield. In the present study, among the parents, the maximum average fruit weight was recorded in Dari Hariharka among the lines and JC-1 among testers. Among all $\mathrm{F}_{1} \mathrm{~s}$, the $\mathrm{F}_{1} \mathrm{SX} \times \mathrm{SM}-6-7$ recorded the highest significant positive heterosis over MP and $\mathrm{BP}$ whereas the $\mathrm{F}_{1} \quad \mathrm{BM} \quad \mathrm{x} \quad \mathrm{JC}-1$ exhibited significant positive heterosis over SP. Similar studies were reported by Suneetha et al., (2008), Nalini et al., (2011), Pachiyappan et al., (2012), Biswas et al., (2013), Makani et al., (2013), Dubey et al., (2014), Reddy and Patel (2014) and Venkata et al., (2014).

The number of fruits per plant directly influences the yield of crop plants. Hence, emphasis should be given to developing such hybrids which bear higher fruits per plant. 
Table.1 Analysis of variance for different characters

\begin{tabular}{|c|c|c|c|c|c|c|c|c|c|c|c|c|c|}
\hline \multirow{2}{*}{$\begin{array}{l}\text { Source of } \\
\text { variation }\end{array}$} & \multirow{2}{*}{$\begin{array}{l}\text { D. } \\
\text { F. }\end{array}$} & \multicolumn{12}{|c|}{ Mean Squares } \\
\hline & & $\begin{array}{c}\text { Days to } \\
50 \% \\
\text { flowering }\end{array}$ & $\begin{array}{c}\text { Days to } \\
50 \% \\
\text { flowering }\end{array}$ & $\begin{array}{l}\text { Plant } \\
\text { height } \\
(\mathrm{cm})\end{array}$ & $\begin{array}{l}\text { Number } \\
\text { of } \\
\text { branches } \\
\text { per plant }\end{array}$ & $\begin{array}{c}\text { Fruit } \\
\text { length } \\
(\mathrm{cm})\end{array}$ & $\begin{array}{l}\text { Fruit } \\
\text { girth } \\
(\mathrm{cm})\end{array}$ & $\begin{array}{c}\text { Number } \\
\text { of fruit/ } \\
\text { plant }\end{array}$ & $\begin{array}{l}\text { Number of } \\
\text { seeds/fruit }\end{array}$ & $\begin{array}{c}\text { Fruit } \\
\text { yield/pl } \\
\text { ant }(\mathrm{kg})\end{array}$ & $\begin{array}{c}\text { Fruit yield/ } \\
\text { ha(q) }\end{array}$ & $\begin{array}{l}\text { Fiber } \\
\text { content } \\
(\%)\end{array}$ & $\begin{array}{l}\text { Shelf } \\
\text { life } \\
\text { (days) }\end{array}$ \\
\hline Replication & 2 & $5.15 * *$ & $5.15 * *$ & 51.49 & 0.66 & 2.91 & 0.57 & 0.74 & 227.75 & 0.01 & 552.41 & 0.06 & 0.39 \\
\hline Genotype & 26 & $39.52 * *$ & $39.52 * *$ & $253.32 * *$ & $41.51 * *$ & $75.65 * *$ & $27.58 * *$ & $29.51 * *$ & $74570.75^{* *}$ & $1.95 * *$ & $96352.24 * *$ & $5.14^{* *}$ & $2.96^{* *}$ \\
\hline Parents & 8 & $33.23 * *$ & $33.23 * *$ & $389.73 * *$ & $4.57 * *$ & $108.13^{* *}$ & $45.63 * *$ & $20.44 * *$ & $72407.54 * *$ & $1.59 * *$ & $79246.69 * *$ & $1.04 * *$ & $5.25 * *$ \\
\hline $\begin{array}{l}\text { Parent vs. } \\
\text { crosses }\end{array}$ & 1 & $364.50 * *$ & $364.50 * *$ & $411.88 * *$ & $701.25^{* *}$ & $12.43 * *$ & $62.63 * *$ & $289.39 * *$ & 871.48 & $22.53 * *$ & $1114851.35^{* *}$ & $11.82 * *$ & $0.72 *$ \\
\hline crosses & 17 & $23.37 * *$ & $23.37 * *$ & $179.80 * *$ & $20.08 * *$ & $64.09 * *$ & $17.03 * *$ & $18.49 * *$ & $79923.98 * *$ & $0.90 * *$ & $44490.20 * *$ & $6.68^{* *}$ & $2.02 * *$ \\
\hline Error & 52 & 2.65 & 2.65 & 16.39 & 1.51 & 1.53 & 0.64 & 0.42 & 434.65 & 0.01 & 260.12 & 0.07 & 0.16 \\
\hline
\end{tabular}


Table.2a Percentage of $\mathrm{F}_{1}$ heterosis over mid-parent (MP), better parent (BP) and standard parent (SP)

\begin{tabular}{|c|c|c|c|c|c|c|c|c|c|}
\hline \multirow[t]{2}{*}{ Genotype } & \multicolumn{3}{|c|}{ Days to 50 per cent flowering } & \multicolumn{3}{|c|}{ Plant height $(\mathrm{cm})$} & \multicolumn{3}{|c|}{ Number of branches per plant } \\
\hline & МPH & ВPH & SPH & МPH & ВPH & SPH & МPH & ВPH & SPH \\
\hline Utsav x JC-1 & $-9.87 * *$ & $-11.03 * *$ & $-11.03 * *$ & -4.32 & $-17.39 * *$ & $-17.39 * *$ & $37.55 * *$ & $27.67 *$ & $49.09 * *$ \\
\hline Utsav x SM-6-7 & $-6.80 * *$ & $-9.43 * *$ & $-11.76 * *$ & $13.05 * *$ & 1.43 & -7.21 & $81.62 * *$ & $75.32 * *$ & $\begin{array}{l}119.99 \\
* *\end{array}$ \\
\hline Utsav x Longai & $-6.57 * *$ & $-7.09 * *$ & $-8.46 * *$ & 5.61 & -6.85 & $-11.40 *$ & $50.12 * *$ & $39.45 * *$ & $62.85 * *$ \\
\hline DH x JC-1 & $-7.12 * *$ & $-8.82 * *$ & $-8.82 * *$ & -2.95 & $-12.39 * *$ & $-12.39 * *$ & $76.98 * *$ & $59.75 * *$ & $98.37 * *$ \\
\hline DH x SM-6-7 & 0.39 & -1.91 & $-5.51 * *$ & 1.21 & -4.84 & $-12.95 * *$ & $111.25 * *$ & $110.15 * *$ & $\begin{array}{l}163.69 \\
* *\end{array}$ \\
\hline DH x Longai & $-6.42 * *$ & $-7.46 * *$ & $-8.82 * *$ & $10.47 * *$ & 2.01 & -2.97 & $108.41 * *$ & $88.28 * *$ & $\begin{array}{l}133.80 \\
* *\end{array}$ \\
\hline MLC-3 x JC-1 & $-5.19 * *$ & $-7.67 * *$ & -2.57 & $-18.04 * *$ & $-22.57 * *$ & $-12.95 * *$ & $58.21 * *$ & $56.80 * *$ & $59.65 * *$ \\
\hline MLC-3 x SM-6-7 & $-6.52 * *$ & $-12.54 * *$ & $-7.72 * *$ & -2.75 & $-11.81 * *$ & -0.85 & $91.74 * *$ & $73.66 * *$ & $\begin{array}{l}117.90 \\
* *\end{array}$ \\
\hline MLC-3 x Longai & $-7.03 * *$ & $-10.10 * *$ & $-5.15 * *$ & -2.19 & $-9.72 *$ & 1.50 & $87.76 * *$ & $86.26 * *$ & $89.63 * *$ \\
\hline MLC-1 x JC-1 & $-3.14 *$ & $-3.68 *$ & $-3.68 *$ & -3.16 & -5.36 & -0.85 & $69.46 * *$ & $55.32 * *$ & $55.32 * *$ \\
\hline MLC-1 x SM-6-7 & $-4.82 * *$ & $-8.18 * *$ & $-9.19 * *$ & $10.03 *$ & 3.05 & 7.96 & $100.18 * *$ & $66.54 * *$ & $\begin{array}{l}108.97 \\
* *\end{array}$ \\
\hline MLC-1 x Longai & $-3.17 *$ & $-3.35 *$ & $-4.41 * *$ & $12.10 * *$ & 6.94 & $12.03 *$ & $88.09 * *$ & $72.25 * *$ & $72.57 * *$ \\
\hline BM x JC-1 & $-6.39 * *$ & $-8.46 * *$ & $-8.46 * *$ & $35.84 * *$ & $15.76 * *$ & $15.76 * *$ & $78.73 * *$ & $53.40 * *$ & $\begin{array}{l}114.09 \\
* *\end{array}$ \\
\hline BM x SM-6-7 & $-3.92 * *$ & $-5.77 * *$ & $-9.93 * *$ & $31.80 * *$ & $16.64 * *$ & 6.70 & $92.39 * *$ & $82.68 * *$ & $\begin{array}{l}154.95 \\
* *\end{array}$ \\
\hline BM x Longai & 2.27 & 0.75 & -0.74 & $17.33 * *$ & 2.11 & -2.87 & $101.24 * *$ & $72.85 * *$ & $\begin{array}{l}141.24 \\
* *\end{array}$ \\
\hline SX x JC-1 & $-5.56 * *$ & $-6.25 * *$ & $-6.25 * *$ & $10.46 * *$ & 6.38 & $14.86 * *$ & $40.44 * *$ & $29.29 *$ & $53.70 * *$ \\
\hline SX x SM-6-7 & $-3.47 *$ & $-6.72 * *$ & $-8.09 * *$ & -1.53 & $-9.06 *$ & -1.80 & $74.28 * *$ & $69.69 * *$ & $\begin{array}{l}112.92 \\
* *\end{array}$ \\
\hline SX x Longai & -1.87 & -1.87 & $-3.31 *$ & $9.56 *$ & 3.04 & $11.25 *$ & $63.03 * *$ & $50.22 * *$ & $78.57 * *$ \\
\hline SEd \pm & 1.15 & 1.33 & 1.33 & 2.86 & 3.31 & 3.31 & 0.87 & 1.00 & 1.00 \\
\hline
\end{tabular}

$* \mathrm{P}<0.05 ; \quad * * \mathrm{P}<0.01$, DH: Dari Hariharka, MLC-1: Manipur local-1, MLC-3: Manipur local-3, BM: Baromohiya and SX: Sagoli Xingiya 
Table.2b Percentage of $\mathrm{F}_{1}$ heterosis over mid parent (MPH), better-parent (BPH) and standard-parent (SPH)

\begin{tabular}{|c|c|c|c|c|c|c|c|c|c|}
\hline \multirow[t]{2}{*}{ Genotype } & \multicolumn{3}{|c|}{ Fruit length $(\mathrm{cm})$} & \multicolumn{3}{|c|}{ Fruit girth (cm) } & \multicolumn{3}{|c|}{ Fruit weight (g) } \\
\hline & МРH & ВPH & SPH & МРH & ВPH & SPH & МРH & ВPH & SPH \\
\hline Utsav x JC-1 & -7.02 & $-14.64 * *$ & $-14.64 * *$ & $-25.47 * *$ & $-28.62 * *$ & $-28.62 * *$ & $-48.18 * *$ & $-57.38 * *$ & $-57.38 * *$ \\
\hline Utsav x SM-6-7 & 2.01 & $-20.32 * *$ & $-33.38 * *$ & $-19.03 * *$ & $-22.94 * *$ & $-29.44 * *$ & $-25.75 * *$ & $-40.54 * *$ & $-61.66 * *$ \\
\hline Utsav x Longai & $-19.01 * *$ & $-22.85 * *$ & $-35.50 * *$ & -5.43 & $-13.91 * *$ & -3.96 & $-16.30 * *$ & $-28.39 * *$ & $-35.06 * *$ \\
\hline DH x JC-1 & $-41.97 * *$ & $-42.39 * *$ & $-41.55 * *$ & -2.47 & $-11.18 * *$ & $-11.18 * *$ & $-38.05 * *$ & $-43.13 * *$ & $-43.13 * *$ \\
\hline DH x SM-6-7 & $42.97 * *$ & 4.61 & 6.13 & 2.45 & 2.09 & $-15.55 * *$ & $12.49 * *$ & $-17.67 * *$ & $-31.15 * *$ \\
\hline DH x Longai & -1.20 & $-13.75 * *$ & $-12.50 *$ & $13.72 * *$ & -1.27 & $10.14 * *$ & $18.34 * *$ & $13.74 * *$ & 3.14 \\
\hline MLC-3 x JC-1 & $-15.92 * *$ & $-26.13 * *$ & $-26.13 * *$ & 1.05 & $-12.38 * *$ & $-12.38 * *$ & $-18.09 * *$ & $-38.91 * *$ & $-38.91 * *$ \\
\hline MLC-3xSM-6-7 & -7.50 & $-25.03 * *$ & $-43.24 * *$ & -2.41 & -7.89 & $-23.80 * *$ & $51.68 * *$ & $35.67 * *$ & $-33.30 * *$ \\
\hline MLC-3 x Longai & $-17.76 * *$ & $-17.78 * *$ & $-37.75 * *$ & -2.40 & $-19.08 * *$ & $-9.73 * *$ & $7.27 *$ & $-17.29 * *$ & $-24.99 * *$ \\
\hline MLC-1 x JC-1 & $-13.29 * *$ & $-23.69 * *$ & $-23.69 * *$ & $11.55 * *$ & $-8.61 *$ & $-8.61 *$ & -1.84 & $-28.23 * *$ & $-28.23 * *$ \\
\hline MLC-1xSM-6-7 & 4.69 & $-15.29 *$ & $-35.60 * *$ & $9.45 *$ & -3.03 & $-19.78 * *$ & $52.24 * *$ & $40.00 * *$ & $-35.29 * *$ \\
\hline MLC-1 x Longai & 6.59 & 6.35 & $-19.15 * *$ & $11.59 * *$ & $-12.27 * *$ & -2.13 & $36.17 * *$ & 2.79 & $-6.79 *$ \\
\hline BM x JC-1 & $28.53 * *$ & $18.20 * *$ & $18.20 * *$ & $6.61 *$ & $-6.69 *$ & $-6.69 *$ & $32.99 * *$ & $8.71 * *$ & $8.71 * *$ \\
\hline BM x SM-6-7 & $57.94 * *$ & $23.20 * *$ & 3.40 & $15.84 * *$ & $10.46 *$ & $-8.62 *$ & $54.90 * *$ & $24.76 * *$ & $-20.78 * *$ \\
\hline BM x Longai & $15.49 * *$ & 9.81 & -7.84 & $18.70 * *$ & -0.72 & $10.75 * *$ & $22.83 * *$ & 4.42 & -5.31 \\
\hline SX x JC-1 & 6.34 & $-10.93 * *$ & $31.92 * *$ & $18.73 * *$ & $-12.94 * *$ & $-12.94 * *$ & $20.20 * *$ & $-15.38 * *$ & $-15.38 * *$ \\
\hline SX x SM-6-7 & -7.29 & $-38.93 * *$ & $-9.55 *$ & $30.68 * *$ & 2.19 & $-15.46 * *$ & $67.95 * *$ & $63.84 * *$ & $-33.18 * *$ \\
\hline SX x Longai & $-14.99 * *$ & $-35.78 * *$ & -4.88 & $36.52 * *$ & -3.19 & $8.00 *$ & $28.84 * *$ & $-6.61 *$ & $-15.31 * *$ \\
\hline SEd \pm & 0.88 & 1.01 & 1.01 & 0.57 & 0.65 & 0.65 & 6.69 & 7.72 & 7.72 \\
\hline
\end{tabular}

$* \mathrm{P}<0.05 ; \quad * * \mathrm{P}<0.01, \mathrm{DH}$ : Dari Hariharka, MLC_1: Manipur local-1, MLC-3: Manipur local-3, BM: Baromohiya and SX: Sagoli Xingiya 
Table.2c Percentage of $\mathrm{F}_{1}$ heterosis over mid parent (MP), better parent (BP) and standard parent (SP)

\begin{tabular}{|c|c|c|c|c|c|c|c|c|c|}
\hline \multirow[t]{2}{*}{ Genotype } & \multicolumn{3}{|c|}{ Number of fruits/plant } & \multicolumn{3}{|c|}{ Number of seeds/fruit } & \multicolumn{3}{|c|}{ Fruit yield/plant (kg) } \\
\hline & МРH & ВPH & SPH & МPH & ВPH & SPH & МРH & ВРH & SPH \\
\hline Utsav x JC-1 & $48.20 * *$ & $35.77 * *$ & $63.13 * *$ & $-4.79 * *$ & $-7.38 * *$ & -2.06 & $-21.80 * *$ & $-30.59 * *$ & $-30.59 * *$ \\
\hline Utsav x SM-6-7 & $51.76 * *$ & $33.41 * *$ & $111.41 * *$ & $-11.51 * *$ & $-27.89 * *$ & $-23.75 * *$ & $16.61 * *$ & 4.60 & $-18.92 * *$ \\
\hline Utsav x Longai & $34.77 * *$ & $29.25 * *$ & $69.16 * *$ & $-31.91 * *$ & $-36.11 * *$ & $-32.44 * *$ & $11.83 * *$ & $-7.56 * *$ & $9.71 * *$ \\
\hline DH x JC-1 & $72.06 * *$ & $68.98 * *$ & $75.26 * *$ & $3.52 *$ & -1.80 & -1.80 & $6.91 * *$ & -0.25 & -0.25 \\
\hline DH x SM-6-7 & $24.77 * *$ & 3.21 & $63.56 * *$ & $28.93 * *$ & $12.30 * *$ & 0.76 & $52.07 * *$ & $30.07 * *$ & $12.65 * *$ \\
\hline DH x Longai & $12.28 * *$ & 0.63 & $31.71 * *$ & $6.01 * *$ & $4.31 *$ & $-3.30 *$ & $32.62 * *$ & $14.70 * *$ & $36.12 * *$ \\
\hline MLC-3 x JC-1 & $88.25 * *$ & $67.86 * *$ & $67.86 * *$ & -1.29 & -2.72 & -2.72 & $47.40 * *$ & 2.58 & 2.58 \\
\hline MLC-3 x SM-6-7 & $55.41 * *$ & $16.11 * *$ & $84.00 * *$ & $25.46 * *$ & $5.74 * *$ & 2.68 & $143.66 * *$ & $99.40 * *$ & $22.73 * *$ \\
\hline MLC-3 x Longai & $38.20 * *$ & $10.46 *$ & $44.56 * *$ & $18.34 * *$ & $15.66 * *$ & $12.31 * *$ & $34.32 * *$ & $-10.66 * *$ & $6.02 * *$ \\
\hline MLC-1 x JC-1 & $85.91 * *$ & $85.04 * *$ & $86.78 * *$ & $-4.11 * *$ & $-8.86 * *$ & 1.15 & $82.24 * *$ & $33.66 * *$ & $33.66 * *$ \\
\hline MLC-1 x SM-6-7 & $45.71 * *$ & $19.26 * *$ & $88.99 * *$ & $-3.86 *$ & $-23.09 * *$ & $-14.65 * *$ & $125.88 * *$ & $98.60 * *$ & $22.24 * *$ \\
\hline MLC-1 x Longai & $20.86 * *$ & 7.04 & $40.09 * *$ & $18.50 * *$ & $8.74 * *$ & $20.68 * *$ & $57.95 * *$ & $10.04 * *$ & $30.59 * *$ \\
\hline BM x JC-1 & $-15.98 * *$ & $-31.22 * *$ & 7.95 & $-5.99 * *$ & $-10.10 * *$ & -1.49 & $17.73 * *$ & $17.44 * *$ & $17.44 * *$ \\
\hline BM x SM-6-7 & $11.47 * *$ & $10.94 * *$ & $75.80 * *$ & $-25.62 * *$ & $-40.21 * *$ & $-34.49 * *$ & $72.85 * *$ & $39.88 * *$ & $39.19 * *$ \\
\hline BM x Longai & $-7.85 *$ & $-15.51 * *$ & $32.61 * *$ & $-3.53 * *$ & $-10.96 * *$ & -2.43 & $15.32 * *$ & $6.00 * *$ & $25.80 * *$ \\
\hline SX x JC-1 & $29.94 * *$ & $9.35 *$ & $60.09 * *$ & $6.53 * *$ & 1.71 & 1.71 & $69.54 * *$ & $35.38 * *$ & $35.38 * *$ \\
\hline SX x SM-6-7 & $37.53 * *$ & $32.29 * *$ & $109.64 * *$ & $12.60 * *$ & -2.48 & $-11.31 * *$ & $131.21 * *$ & $127.74 * *$ & $40.17 * *$ \\
\hline SX x Longai & $16.15 * *$ & $9.99 *$ & $61.03 * *$ & $16.48 * *$ & $15.38 * *$ & $6.96 * *$ & $53.17 * *$ & $15.11 * *$ & $36.61 * *$ \\
\hline SEd \pm & 0.46 & 0.53 & 0.53 & 14.74 & 17.02 & 17.02 & 0.05 & 0.06 & 0.06 \\
\hline
\end{tabular}

*P<0.05; ${ }^{* *} \mathrm{P}<0.01$, DH: Dari Hariharka, MLC_1: Manipur local-1, MLC-3: Manipur local-3, BM: Baromohiya and SX: Sagoli Xingiya 
Table.2d Percentage of $\mathrm{F}_{1}$ Heterosis over mid parent (MP), Better parent (BP) and Standard parent (SP)

\begin{tabular}{|l|l|l|l|l|l|l|}
\hline \multirow{2}{*}{ Genotype } & \multicolumn{3}{c|}{ Fiber content (\%) } & \multicolumn{3}{c|}{ Shelf life (days) } \\
\cline { 2 - 7 } & \multicolumn{2}{|c|}{ MPH } & \multicolumn{1}{c|}{ BPH } & \multicolumn{1}{c|}{ SH } & \multicolumn{1}{c|}{ BPH } \\
\hline Utsav x JC-1 & 7.48 & 4.07 & $11.12 *$ & $9.22 *$ & -4.96 & $28.38 * *$ \\
\hline Utsav x SM-6-7 & 6.30 & -0.42 & $21.72 * *$ & -3.75 & $-11.12 * *$ & $20.07 * *$ \\
\hline Utsav x Longai & 4.05 & -1.06 & $17.16 * *$ & -4.35 & $-18.53 * *$ & $10.06 *$ \\
\hline DH x JC-1 & $18.10 * *$ & 5.11 & $34.76 * *$ & $-9.66 *$ & $-12.52 *$ & -6.61 \\
\hline DH x SM-6-7 & $-18.49 * *$ & $-20.39 * *$ & 2.06 & $8.58 *$ & 4.94 & $20.07 * *$ \\
\hline DH x Longai & $8.93 * *$ & 4.77 & $34.32 * *$ & 5.8 & 0.00 & 6.76 \\
\hline MLC-3 x JC-1 & $15.10 * *$ & 7.22 & 7.22 & 0.44 & -2.05 & -2.05 \\
\hline MLC-3 x SM-6-7 & $13.91 * *$ & -2.83 & $18.78 * *$ & $27.36 * *$ & $16.58 * *$ & $33.38 * *$ \\
\hline MLC-3 x Longai & -6.62 & $-19.28 * *$ & -4.42 & 7.06 & 7.06 & 1.75 \\
\hline MLC-1 x JC-1 & 0.66 & -4.13 & 5.96 & -5.6 & $-14.32 * *$ & 5.11 \\
\hline MLC-1 x SM-6-7 & $-12.50 * *$ & $-16.69 * *$ & 1.84 & 3.61 & 0.12 & $22.82 * *$ \\
\hline MLC-1 x Longai & $12.58 * *$ & $8.83 *$ & $28.87 * *$ & 4.00 & -7.71 & $13.21 *$ \\
\hline BM x JC-1 & $89.66 * *$ & $79.28 * *$ & $101.33 * *$ & $-10.65 * *$ & $-25.55 * *$ & $11.71 *$ \\
\hline BM x SM-6-7 & $88.45 * *$ & $80.78 * *$ & $120.99 * *$ & 5.45 & $-7.07 *$ & $39.44 * *$ \\
\hline BM x Longai & $30.67 * *$ & $27.30 * *$ & $50.74 * *$ & 2.06 & $-16.64 * *$ & $25.08 * *$ \\
\hline SX x JC-1 & $14.91 * *$ & 4.46 & $27.69 * *$ & $23.53 * *$ & $20.07 * *$ & $20.07 * *$ \\
\hline SX x SM-6-7 & $8.61 *$ & $8.61 *$ & $32.77 * *$ & $17.45 * *$ & 7.17 & $22.62 * *$ \\
\hline SX x Longai & 2.45 & 0.84 & $23.27 * *$ & $15.03 * *$ & $14.64 * *$ & 8.96 \\
\hline \multicolumn{1}{|c|}{ SEd \pm} & 0.18 & 0.21 & 0.21 & 0.29 & 0.33 & 0.33 \\
\hline
\end{tabular}

*P< $<0.05 ; \quad * * \mathrm{P}<0.01$, DH: Dari Hariharka, MLC_1: Manipur local-1, MLC-3: Manipur local-3, BM: Baromohiya and SX: Sagoli Xingiya 
Table.3 Promising crosses for fruit yield and component characters showing significant standard parent heterosis

\begin{tabular}{|c|c|c|c|}
\hline $\begin{array}{c}\text { Most heterotic } \\
\text { crosses }\end{array}$ & $\begin{array}{c}\text { Mean fruit } \\
\text { yield/plant } \\
\mathbf{( k g})\end{array}$ & $\begin{array}{c}\text { Heterosis \% } \\
\text { for yield over } \\
\text { standard } \\
\text { parent }\end{array}$ & $\begin{array}{c}\text { Significant standard parent heterosis } \\
\text { for other traits in desirable direction }\end{array}$ \\
\hline SX x SM-6-7 & 3.80 & $40.17 * *$ & FYP, NSF, NFP, FC, SL, DF, PH and \\
NBP.
\end{tabular}

FYP: Fruit yield per plant (kg), NSF: Number of seeds per fruit, NFP: Number of fruits per plant, FC: Fiber content (\%), SL: Shelf life of fruit (days), DF: Days to 50\% flowering, PH: Plant height (cm), FL: Fruit length (cm), FG: Fruit girth (cm), NFP: Number of fruits per plant, NBP: Number of branches per plant, DH: Dari Hariharka, MLC-1: Manipur local-1, BM: Baromohiya and SX: Sagoli Xingiya

The improvement in the number of fruits per plant can be achieved by using diverse parents and progenies. From the present study, MLC-3 x JC-1, MLC-1 x JC-1 and Utsav x SM-6-7 were found to have the highest significant positive heterosis over MP, BP and SP compared to all the other cross combinations. Similar results were also found by earlier works like Shafeeq et al., (2007), Chowdhury et al., (2010) and Biswas et al., (2013), Reddy and Patel (2014), Venkata et al., (2014) and Desai et al., (2016).

Brinjal is regarded as poor men's vegetable. Higher yield is necessary to meet the people's demand for vegetables which will essentially provide valuable minerals and nutrient for proper growth, development and human health. Higher yield is also necessary to improve the economic status of Indian farmers for the overall benefit of the country's economy. In the present study, a highly significant positive heterosis (MP and $\mathrm{BP}$ ) was exhibited by the $\mathrm{F}_{1} \mathrm{SX} \times \mathrm{SM}-6-7$ followed by MLC-3 x SM-6-7 which exhibited significant heterosis (SP) for yield per plant and yield per ha. Similar findings were reported by several workers such as Kumar et al., (2012), Biswas et al., (2013), Makani et al., (2013), Dubey et al., (2014), Reddy and Patel (2014), Venkata et al., (2014) and Boddepalli et al., (2016).

The seed inside the fruit is a major constraint for consumer preference hence the development of variety having few seeds inside the fruit is desirable for consumption. In the present investigation, many hybrids having lesser seed over their parents were observed. The hybrid Utsav $\mathrm{x}$ Longai recorded to have highly significant negative heterosis for this trait over MP while the $\mathrm{F}_{1} \mathrm{BM} \times \mathrm{SM}-6-7$ was found to have highly significant negative heterosis over both BP and SP. High yielding hybrids with fewer seeds per fruit but with a seed parent having more number of seeds per fruit will be preferred in a commercial hybrid 
seed production. Only high yielding hybrid never full fills the consumer requirement and producer requirement. Hence the quality of produce is a prerequisite for the fulfilment of both consumer and producer requirement.

Keeping this point in view, high fibre content and long shelf life is a necessity for hybrid development. In the present study, the $\mathrm{F}_{1} \mathrm{BM}$ $\mathrm{x}$ JC-1 showed highly significant positive heterosis over MP whereas BM x SM-6-7 had highly significant positive heterotic effect over both BP and SP for high fibre content which could fulfil the consumer and farmers requirement. For shelf life which prevents the market glut, the $\mathrm{F}_{1 \mathrm{~S}}$ MLC-3 x SM-6-7 and SX $x$ JC-1 were found to have a longer shelf life over MP and BP. The $\mathrm{F}_{1}$ BM x SM-6-7 was found with highly significant positive heterosis over SP (JC- 1) for both the traits. These crosses can be exploited for developing high-quality hybrids.

On the basis of economic heterosis, it can be concluded that the heterosis breeding could be advantageous for the improvement of brinjal genotypes for yield and fruit characters. The $\mathrm{F}_{1}$ s namely, SX x SM-6-7 and BM x SM-6-7 showing significant standard parent heterosis (SPH) in Table 2a, 2b, 2c and 2d were probably due to the highly significant heterotic effect in yield and its contributing traits viz., number of branches per plant and number of fruits per pant which can be utilized for commercial exploitation of heterosis towards maximizing yield.

\section{Acknowledgements}

The authors acknowledge the help received from scientists of AICRP Scheme on Vegetable Crops, AAU, Jorhat and the Directorate of Post Graduate Studies, Assam Agricultural University, Jorhat, Assam with thanks for providing necessary facilities during the course of the investigation.

\section{References}

Biswas, L.; Mehta, N. and Ansari, S.F. 2013. Hybrid vigour studies in Brinjal (Solanum melongena L.). Glob. J. Sci. Front. Res. Agric. Veter., 13(9).

Boddepalli, V.N.; Dubey, A.K. and Dabbas, M.R. 2016. Studies of standard heterosis for quantitative traits in eggplant (Solanum melongena L.). Intern. J. Agril. Sci., 12: 38-41.

Chowdhury, M.J.; Ahmad, S.; Nazim, U.M.; Quamruzzaman, A.K.M. and Patwary, M.A. 2010. Expression of Heterosis for productive traits in F1 brinjal (Solanum melongena L.). hybrids. The Agriculturists, 8: 8-13.

Desai, K.M.; Saravaiya, S.N.; Patel, A.I.; Tank, R.V. and Patel, D.A. 2016. Heterosis for yield and its relevant characters in brinjal (Solanum melongena L.). Adv. Life Sci., 5: 5356-5359.

Dudhat, N.M.; Saaliya, J.J.; Sharma, L.K. and Kelalya, D.S. 2013. Heterosis for earliness and plant stature in brinjal (Solanum melongena L.). Soc. Sci. Dev. Agric. Tech. Progr. Res., 8: 555559.

Joshi, N.; Singh, Y.V. and Maurya, S.K. 2008. Combining ability analysis for various quantitative traits in eggplant (Solanum melongena L.). Ann. Hort., 1: 32-36.

Kamalakkanan, T.; Karuppaiah, P.; Sekar, K. and Senthilkumar, P. 2007. Line $\mathrm{x}$ tester analysis in brinjal for yield and shoot and fruit borer tolerance. Indian J. Hort., 64: 420-424.

Kumar, A.; Kumar, P.; Singh, A. and Kumar, S. 2012. Status of combining ability in relation to other genetic parameters in brinjal (Solanum melongena L.) TECHNOFAME- A J. Multidisc. Adv. Res., 1: 71-82.

Makani, A.Y.; Patel, A.L.; Bhatt, M.M. and Patel, P.C. 2013. Heterosis for yield 
and its contributing attributes in brinjal (Solanum melongena L.). The Bioscan, 8: 1369-1371.

Murugan, AM.; Singh. R. A. and S. Vidhya 2011. Evaluation of shelf life and organoleptic aspect of fruits stored in a modified traditional earthen pot cool chamber, Ind. J. of traditional knowledge, 10: 375-379.

Nalini, A.D.; Patil, S.A. and Salimath, P.M. 2011. Heterosis and combining ability analysis for productivity traits in brinjal (Solanum melongena L.). Karnataka J. Agril. Sci., 24 : 622-625.

Reddy, E.E.P. and Patel, A.I. 2014. Heterosis studies for yield and yield attributing characters in brinjal (Solanum melongena L.). J. Recent Adv. Agric., 2: 175-180.

Sadasivam, S. and A. Manickam 1992. Biochemical Methods for Agricultural Sciences. Wiley Eastern Ltd., New Delhi.

Shafeeq, A. 2005. Heterosis and combining ability studies in brinjal (Solanum melongena L.) Unpublished M. Sc. Thesis. University of Agricultural Sciences, Dharwad, Karnataka, India. Shafeeq, A.; Madhusudan, K.; Hanchinal,
R.R.; Vijayakumar, A.G. and Salimath, P.M. 2007. Heterosis in Brinjal. Karnataka J. Agril. Sci., 20: 33-40.

Sharma, V.K. 2010. Manifestation of heterosis for quantitative traits in bacterial wilt resistant lines of brinjal (Solanum melongena L). J. Hill Agric., 1: 193196.

Singh, K.; Sidhu, A.S. and Kumar, A. 2012. Heterosis for fruit yield and its components in brinjal (Solanum melongena L.). J. Hort. Sci., 7: 142144

Suneetha, Y.; Kathiria, K.B.; Patel, J.S. and Srinivas, T. 2008. Studies on heterosis and combining ability in late summer brinjal. Indian J. Agril. Res., 42: 171176.

Venkata, N.B.; Dubey, A.K.; Tiwari, P.K. and Dabbas, M.R. 2014. Line $\mathrm{x}$ Tester analysis for yield components and Cercospora leaf spot resistance in brinjal (Solanum melongena L.). Electr. J. Plant Breed., 5: 230-235.

Venkata, N.B.; Dubey, A.K.; Tiwari, P.K. and Shukla, I.N. 2015. Combining ability studies for yield and its attributing traits in brinjal (Solanum melongena L.). Curr. Adv. Agril. Sci., 7: 117-119.

\section{How to cite this article:}

Niranjan Kumar Chaurasia, Prabalee Sarmah, Nagendra Sarma Barua, Debojit Sarma, Hiramani Barman and Purna Kanta Barua. 2019. Heterotic Effect of Some Intra-specific Crosses of Brinjal (Solanum melongena L.) for Yield and Yield Attributes. Int.J.Curr.Microbiol.App.Sci. 8(12): 2913-2923. doi: https://doi.org/10.20546/ijcmas.2019.812.338 